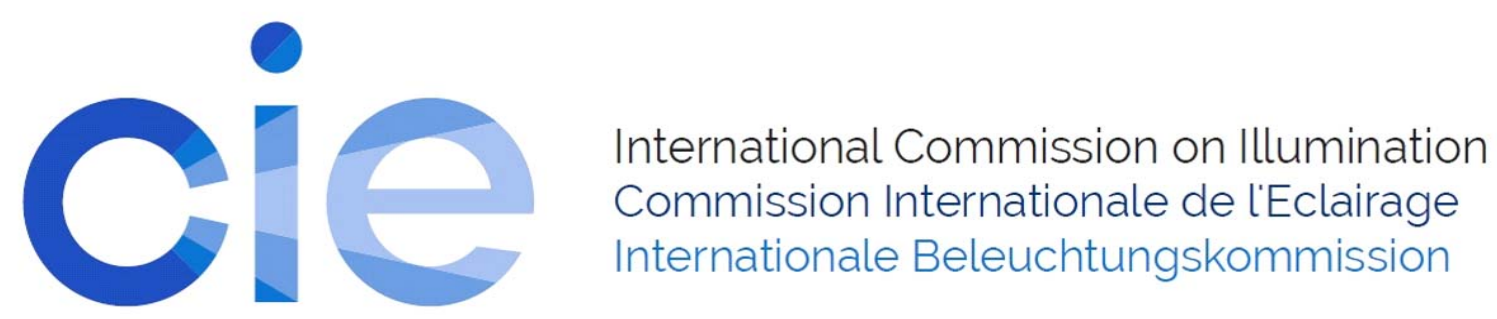

OP74

\title{
INFLUENCE OF MATERIAL CHARACTERIZATION IN THE DESIGN OF TUNNEL LIGHTING INSTALLATIONS
}

\author{
Paola lacomussi et al.
}

DOI 10.25039/x46.2019.OP74

from

CIE x046:2019

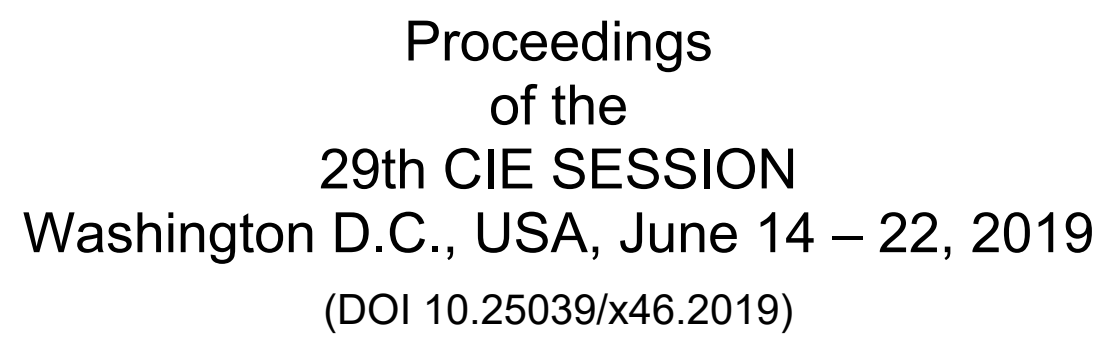

The paper has been presented at the 29th CIE Session, Washington D.C., USA, June 14-22, 2019. It has not been peer-reviewed by CIE.

(C) CIE 2019

All rights reserved. Unless otherwise specified, no part of this publication may be reproduced or utilized in any form or by any means, electronic or mechanical, including photocopying and microfilm, without permission in writing from CIE Central Bureau at the address below. Any mention of organizations or products does not imply endorsement by the CIE.

This paper is made available open access for individual use. However, in all other cases all rights are reserved unless explicit permission is sought from and given by the CIE.

CIE Central Bureau

Babenbergerstrasse 9

A-1010 Vienna

Austria

Tel.: +4317143187

e-mail: ciecb@cie.co.at

www.cie.co.at 


\title{
INFLUENCE OF MATERIAL CHARACTERIZATION IN THE DESIGN OF TUNNEL LIGHTING INSTALLATIONS
}

\author{
lacomussi P. ${ }^{1}$, Rossi G. ${ }^{1}$ \\ 1 INRIM, Istituto Nazionale di Ricerca Metrologica, Torino, ITALY \\ p.iacomussi@inrim.it
}

DOI 10.25039/x46.2019.OP74

\begin{abstract}
The paper describes the influence of the characterisation of reflectance behaviour of tunnel pavements and wall materials on the tunnel lighting design. CIE 189 document suggests considering lambertian behaviour for inter-reflection calculations for road luminance evaluation at design stage, because, unfortunately, no bi-directional reflection data for tunnel surfaces are commonly available. This simplification is supported by the low impact of interreflection contribution to road luminance. A European funded research project ha the task of developing the metrological support for the road surface characterisation in new geometries of measurements. The paper suggests to apply the outcomes on new geometries to tunnel wall materials characterisation suggesting that the suggested SURFACE observation angle of $2.29^{\circ}$ can be useful for short tunnel too, including wall surfaces.
\end{abstract}

Keywords: Road tunnel lighting, energy savings, SURFACE, EMPIR, luminance coefficient, rtables.

\section{Introduction}

The luminance coefficient is an essential parameter for the design of efficient and safe road lighting installations according to luminance criteria: the road must have average values of luminance and luminance uniformities, to assure safety for road users, regardless if it is an open road or a tunnel road. Indeed in tunnel lighting some additional parameters are required too, like the wall luminance, but are still based on luminance.

Usually, road luminance values required in standards and CIE Technical report (CIE 2010a) (CIE 2010b) (EN 2015a to b) are obtained through calculations, considering the luminous intensity distributions of luminaires and the pavement reflective properties as tabulated, only for a few selected geometrical conditions, in reference tables published inside standards or in the CIE 144 (CIE 2001) technical report, a de facto standard.

Regarding tunnel lighting, currently no European Standard is available and CIE technical reports (CIE 2010 a) (CIE 2004) are used as the facto standard.

The design of tunnel lighting requires to different approaches, one for daylight conditions and one for night-time. The second condition can follows the rules and requirements for road lighting but standards generally requires an higher value of the average road luminance because un incident in a tunnel is supposed to be more danger then a incident, in the same condition but in a normal road. The first one is more complex because shall consider that when the driver is looking at the first part of the tunnel (i.e. the threshold zone) he/she is approaching the tunnel (i.e. he/she is in the access zone) and the external environmental light create a high level of glare (the black hole effect). For this reason the luminance level in the threshold zone can be $100-200$ times higher than the maximum level standard require for road lighting on the same type of road.

Three other aspects shall be considered as peculiarities of tunnel lighting:

- The lighting installation is working, at different lighting level, but for $24 \mathrm{~h}$ per day, so the energy consumption and luminaire maintenance becomes an important cost contribution to the tunnel management 
- The tunnel is a close environment completely managed by a single responsible, so glare conditions, influences of other installation are completely under control.

- The walls, if adequality bright, can improve the perception of danger object on the carriageway and of other vehicles, can create a sort of optical guide to better understand the road layout, can reduce the sense of claustrophobia, improve the driver comfort and considering the reflected light increase the luminance level and uniformities on the carriageway.

Tunnel lighting is a specific application of road lighting, with its own peculiarities because tunnel environment is very far from open road situation. In particular, additional aspects like:

- internal inter-reflections (walls and ceilings),

- Luminaire spacing and output variables along the tunnel length,

- different lighting geometries with also a reduced height of installation of luminaires,

- different viewing geometries with calculation fields longer than for open roads,

- variability on the visual requirements because driver visual adaptation variability along the tunnel length.

CIE publication 189 (CIE 2010a) suggests several additional quality parameters for tunnel lighting only to consider in design stage but also for on site verification, like:

- average wall and road luminance, longitudinal and overal uniformity, for zones at constant lighting levels

- transverse average luminance of walls and road,

- average and minimum values of revealing coefficient,

- threshold increment.

For the evaluation of the above parameters it is necessary to calculate, and measure on site the following quantities:

- road luminance and illuminance,

- wall luminance and illuminance at given heights

The CIE document also recognizes that, unfortunately, no bi-directional reflection (BRDF) data for tunnel surfaces are commonly available nor recommended calculation procedure and suggests to consider lambertian behaviour for wall luminance and inter-reflection calculations, because the inter-reflection contribution to road and wall luminances is one order of magnitude lower than direct component of luminaires. But it is really important to highlight that for the calculation of the luminance comings from the direct luminaire components the knowledge of the luminance coefficient of surface is unavoidable. A lambertian behaviour is also suggested for the road surface if the r-table of the installed pavements is not know, calculating the mean road reflectance value from the ration between the luminance and illuminance on the pavement surface.

The lambertian generalisation brings to low reliability of the calculated values of tunnel lighting: discrepancies between calculated and measured values can bring to not compliance of lighting system in extreme cases. This arrives when generalisations are applied both to wall and pavement behaviour. Tunnel walls, especially current materials, do not have a lambertian behaviour as supposed: BRDF measurements on clean tiles showed specular peak added to a lambertian distribution (Figure 1). LED lighting fixtures having very sharp luminous intensity distribution highlight this peculiarity. The second contribution is related to pavement luminance coefficient that is used for calculating the expected road luminance. The use of CIE144 r-tables (CIE 2004) brings to the aforesaid discrepancies in road luminance because the low representativeness of current road surfaces. Indeed these reference tables provide values based on measurements performed more than 40 years ago and not for all the geometrical conditions necessary, like those requested in the European standard (EN 2015 b). This problem is well known to the lighting community and to overcome the problem designers usually over-estimate the installed flux. An European research project (Surface 
2019) funded by EURAMET (the association of European Metrological Institutes) (Euramet 2019) with the EMPIR programme, has the task of providing new optimized geometries and traceable data of road luminance coefficient, as contribution efficient and smart road lighting.

\section{Materials and Methods}

The aims of CIE 189 (CIE 2010a) is to provide common rules for the calculation of the quality parameters of tunnel lighting installations, because lighting installations are designed using different methods all with the purpose of calculating road luminance and based on two different CIE documents on CIE 140 (CIE 2019) and CIE 88 (CIE 2004).

Because tunnel are partly closed volumes, inter-reflection between walls and road surfaces must be taken in account too. A generalisation of the reflectance behaviour of both lit surfaces, horizontal and vertical ones is one of the main sources of discrepancies between measured and designed data.

Regarding vertical lit surfaces the applied simplification regards the reflectance spatial behaviour considering them following the Lambertian law. For horizontal surfaces, the road pavement, the simplification is in using r-tables not representative of actual road surface nor of viewing and lighting geometries.

\subsection{Vertical tunnel surfaces discrepancies}

CIE 189 (CIE 2010 a) states, that the low reliability of tunnel lighting calculations of photometric data and quality criteria is due to the lack of knowledge of the reflection properties of the road surface and walls required for the evaluation of interreflection. The document suggests some simplified assumptions $\square$ regarding the reflectance behaviour that should increase the reliability:

- To consider tunnel vertical surfaces having a diffuse reflections according to Lambert's law;

- Luminance (and illuminance) of road and walls should be calculated as the results of two different components:

- Direct illumination from the luminaieres;

- Indirect illumination due to inter reflections;

- CIE 189 states that inter reflections contributions are one order of magnitude lower than direct illumination, so the calculation can be stopped at the first order, considering the first lit surface as secondary source to light the next surface, with just two levels of reflections: the first from walls to the road and the second between walls and then to road.

This last point is about walls inter-reflections contribution to luminance, but when considering calculations from the direct component of luminaire of the road as well of the wall luminance and illuminance the simplification of using Lambertian behaviour can bring higher discrepancies. This has effects also on the contrast revealing coefficient $q_{c}$, the ratio among road luminance and vertical illuminance on a specific position, a parameter necessary for establishing the threashold luminance (CIE 2004):

$$
q_{c}=\frac{L}{E_{v}}
$$

where:

$L$ is the road luminance

$E_{v}$ the vertical illuminance.

Figure 1 shows the BRDF values on the azimuth planes $\varphi 2=\left(0,180^{\circ}\right)$ for a polar incidence of $15^{\circ}, 30^{\circ}$ of a typical tunnel tile goniophotometrically measured at the INRIM goniometer 
(lacomussi 2015), given the reference angular system shown in Figure 2. It is clear that this sample can be hardly considered with a lambertian behaviour.

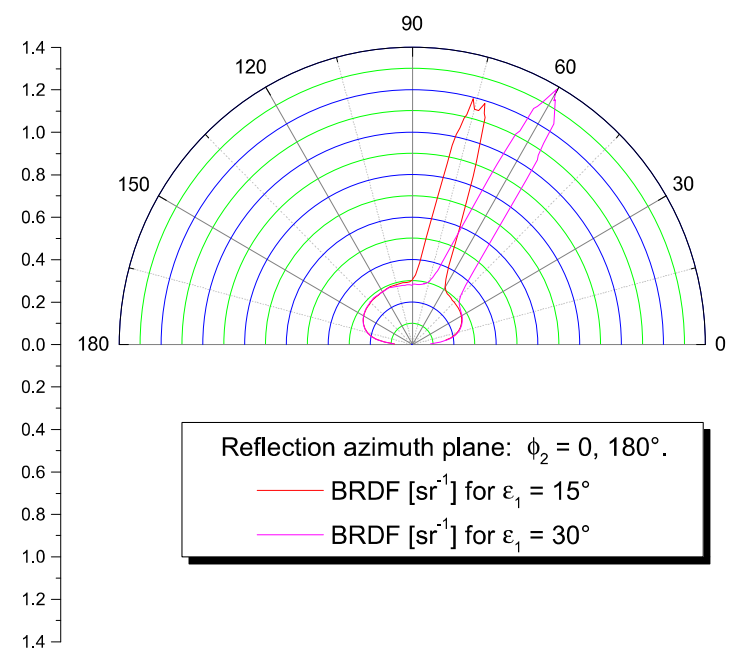

Figure $1-B R D F$ values on the azimuth planes $\varphi 2=\left(0,180^{\circ}\right)$ for a polar incidence of $15^{\circ}, 30^{\circ}$

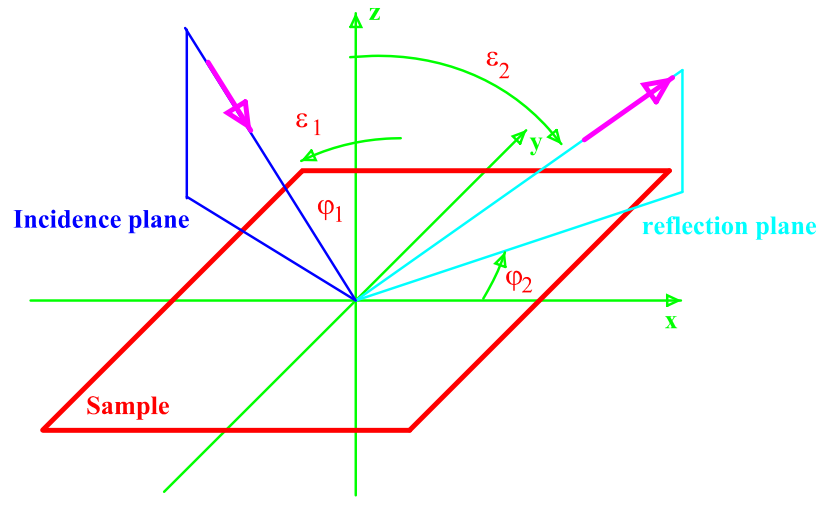

Figure 2 - Reference geometrical system for BRDF measurements at the INRIM goniometer.

It is true that a deep knowledge of BRDF of materials installed on walls can led to higher accuracy results if used in a scientific simulation software able to manage BRDF distribution. To lower calculations impact, reaching the same accuracy of the results, it is not necessary a full BRDF characterization: like for road lighting calculations, the knowledge of pavement BRDF for few selected directions of illumination and observations is enough for lighting calculations, as the r-tables are a simplified descriptors of pavements BRDF.

\subsection{Pavement reflectance discrepancies}

It is well known that the current available reference data tabulated in CIE r-tables of CIE 88 (CIE 2004) are not representative of current pavements because rely on measurements made in the seventies and in some cases can lead to discrepancies on average, between designed and actual luminance values often over $30 \%$ and sometimes over $50 \%$ (Chain 2007). This is because pavements tabulated reflectance data are no more representative of actual road surfaces (Dumont 2007) (Fotios 2006) (Ylinen 2010) and these influences can be more relevant with SSL luminaires having .

Road luminance is calculated from the formula in (EN 2015b) that describes the relationship between the road luminance and illuminance calculated for every direction of illumination $(\varepsilon$, $\beta)$ (Figure 4 of (EN 2015b)) that provides the "Angular relationships for luminaire at tilt during measurement, observer, and point of observation", considering the luminance coefficient, expressed as r-table, of the installed road surfaces. It is well know that available published $r$ values are no more representative of actual road surfaces. Two different and independent 
studies (Gidlund 2019) (Salata 2015) show the relevant advantages in using bright pavements.

Indeed (Gidlund 2019) highlights discrepancies in energy consumptions, when the design is based on CIE r-tables or on measured r-tables of actual pavements. The research highlighted that when the installed actual pavement is significantly different from the one used in calculations with differences on both diffuse $\left(Q_{0}\right)$ and specular components $\left(S_{1}\right)$ of the luminance coefficient (q), the discrepancies between designed and installed values can be relevant and standard compliance cannot be assured. Only a smart lighting system can assure the compliance with the normative values checking the actual luminance values and arranging the luminaires output according. Moreover, when smart systems are associated with bright pavements, large energy savings can be obtained in open road, with savings higher than $40 \%$ on annual base. Luckily road tunnels usually have this kind of controllers installed to measure and arrange according especially entrance luminance. The same results on bright pavements are confirmed for tunnel lighting too (Salata 2015) including the same energy savings: two different lighting installations, providing the same luminance levels in a tunnel, can large energy discrepancies. Calculations for bright pavements bring to energy savings higher than $40 \%$ when associated with LED lighting system, instead of a tunnel lighting system designed with a dark CIE reference pavement.

SURFACE project has also the task of developing metrology research to support road lighting standardisation, providing reference data of current road surfaces, optimized reference geometrical conditions and developing initial stage of on site measuring instrument able to measure pavement characteristics also in the new geometries that will be selected by the project.

SURFACE is proposing a different geometry for the measurement of q luminance coefficient of pavements to recognize the differences in viewing angles in extra-urban and urban environments. It is clear that in urban environment $1^{\circ}$ of observation and subsequent very long viewing distances $(>85 \mathrm{~m})$ are unrealistic. For this reason the project suggests an additional observation angle of $2.29^{\circ}$, the same viewing condition used in road marking measurements. Regarding illumination angles the project is working also on the extend $\mathrm{r}$ tables of (EN 2015b) for low mounting luminaires.

\subsection{Impact of SURFACE outcomes on tunnel lighting}

For short or mountain tunnels, the condition of observation $1^{\circ}$ as in reference standard (EN 2015b) (CIE 2004) (CIE 2010a) (CIE 201b) can be useless because the observation distance is unsuitable to represent driver conditions of observation for the tunnel length or turn. In this framework, the SURFACE proposal of an additional observer at $2.29^{\circ}$ to be used in urban environment can be suitable and efficient for short tunnels too. As well the discussion is ongoing on the use of geometries represented in the extended r-table of (EN 2015b) for low mounting luminaires can be suitable for use in tunnel too because the mounting height of tunnel luminaires.

An open question remains on the most suitable geometries for reflectance measurement of the materials used on the tunnel walls. The measurement of Figure 1 clearly demonstrated that the simplification of lambertian behaviour is not suitable for describing the BRDF distribution of some tunnel walls materials. If the lambertian generalisation is suitable for inter-reflection calculations, because the low impact respect the direct illumination from luminaires, this is not true for the calculations of expected wall luminance and illuminance from direct luminaires component. A better description of spatial distribution of the reflectance (luminance coefficient will be suitable) is needed, but the choice of the conditions of measurements (e.g. the geometries) should be based on additional simple principles to the obvious usefulness for lighting calculations:

- convenience of industrial and laboratory level instrumentations;

- availability of metrological chain, for assuring traceability of the measurements;

- consistency of already available measurements conditions of already established metrological chain. 
All previous conditions are met if the suggested SURFACE geometries are applied to the characterisation of tunnel walls materials, (with the appropriate tilt). Indeed, SURFACE will not only develop new portable instruments able to measure in the $2.29^{\circ}$ of observation, but also the metrological traceability chain. Considering a typical tunnel dimension of $9 \mathrm{~m}, 2$ lanes, the observation angle of $2.29^{\circ}$ corresponds to a viewing distance on the wall of about $55 \mathrm{~m}$ (for an observer in the middle of a carriageway): a distance suitable for short tunnel applications too. Moreover, if the same approach of road luminance coefficient characterisation is applied to walls, the additional $Q_{0}$ and $S_{1}$ parameters, describing the diffuse and specular reflectance properties, can be usefully applied in the aforesaid simplification of inter-reflection contributions, providing upper and lower behaviour boundaries.

\section{Conclusions}

The use of full measured BRDF values of vertical surfaces in tunnel lighting design is not realistic due the lack of database with measured values and calculations load. Moreover, (CIE 2010a) suggests for calculations simplification to consider a lambertian reflectance wall behaviour. Besides the main discrepancies between designed and installed road luminance values it is proven in literature that arrive from the low reliability of r-tables used in calculations. The funded research project SURFACE has the task to provide the metrological support to standard developing organization, suggest new geometries for luminance coefficient measurements, reference data of current pavements and instrument prototype for on site characterisation in the new measurement geometries. The paper suggests to apply the SURFACE measurement geometries also for tunnel wall reflectance characterisation, to increase reliability of wall luminance calculations. The suggestion will be tested inside the SURFACE actions including its reliability and application limits to short or mountain tunnels.

\section{Acknowledgement}

This work is partially covered by project "16NRM02 Surface, Pavement surface characterisation for smart and efficient road lighting" that has received funding from the EMPIR programme. EMPIR programme is co-financed by the Participating States and from the European Union's Horizon 2020 research and innovation programme.

\section{References}

CHAIN, C. Lopez, F. et al. 2007. Impact of real road photometry on public lighting design. In Proc. Of the CIE 26th Session, Beijing, China, 4-11 July 2007; The International Commission on Illumination, Vienna: CIE

CIE 2001 CIE144:2001. Road Surface and Road Marking Reflection Characteristics; Technical Report. Vienna: CIE.

CIE 2004. CIE 88:2004. Guide for the Lighting of Road Tunnels and Underpasses. Vienna: CIE.

CIE 2010a CIE189:2010. Calculation of tunnel lighting quality criteria; Technical Report; Vienna: CIE.

CIE 2010b CIE115:2010. Lighting of Roads for Motor and Pedestrian Traffic; Technical Report; Vienna: CIE.

CIE 2011. CIE 194:2011. On Site Measurement of the Photometric Properties of Road and Tunnel Lighting. Vienna: CIE.

CIE 2019. CIE 140:2019 Road lighting calculations. Vienna: CIE.

DUMONT, E. Paumier, J. Are standard tables $\mathrm{R}$ still representative of the properties of road surfaces in France? In Proc. Of the CIE 26th Session, Beijing, China, 4-11 July 2007; The International Commission on Illumination, Vienna: CIE.

EN 2015a EN 13201-2:2015. Part 2: Performance requirements. In Road Lighting; CEN: Bruxelles: CEN. 
EN 2015b EN 13201-3:2015. Part 3: Calculation of performance. In Road Lighting; CEN: Bruxelles: CEN.

EN 2015c EN 13201-4:2015. Part 4: Verification of performance. In Road Lighting; CEN: Bruxelles: CEN.

EN 2015d EN 13201-5:2015. Part 5: Energy performance indicators. In Road Lighting; CEN: Bruxelles: CEN.

EURAMET 2019. The European Association of National Metrology Institutes. Available online: https://www. euramet.org/about-euramet/ (accessed on 28 March 2019).

FOTIOS, S. 2006; Boyce, P.; Ellis, C. The effect of pavement material on road lighting performance. Light. J. Rugby, 71, 35.

GIDLUND, H. 2019 et al., Road Surface Photometric Characterisation and Its Impact on Energy Savings, Coatings, 9(5), 286; doi:10.3390/coatings9050286

IACOMUSSI, P. 2015, Radis, M., Rossi, G., Goniometric and colorimetric properties of paints and varnish appearance, Proc. SPIE 9398 Measuring, Modeling and Reproducing Material Appearance, San Francisco: SPIE

SALATA, F., 2015, et al., Energy optimization of road tunnel lighting systems. Sustainability, 7.7: 9664-9680; doi:10.3390/su7079664.

SURFACE 2019 Surface Pavement Surface characterisation for smart and efficient road lighting. Available online: https: //surface-nrm02.eu (accessed on 28 March 2019).

UNI 2011. UNI 11095:2011. Road tunnel lighting. Milano: UNI (in Italian).

YLINEN A. 2010; Puolakka, M.; Halonen, L. Road surface reflection properties and applicability of the r-tables for today's pavement materials in Finland. Light \& Engineering 2010, 18. 